\title{
Adquisición de Señales Ambientales para un Sistema de Alerta Temprana
}

\author{
Jordan D. Guillot ${ }^{(1,2)}$, Carlos A. Robles ${ }^{(2)^{*}}$ y Jesús D. Callejas ${ }^{(1)}$ \\ (1) Facultad de Ingeniería, Universidad Cooperativa de Colombia. Troncal del Caribe, Sector Mamatoco, \\ Santa Marta-Colombia \\ (2) Facultad de Ingeniería, Universidad del Magdalena. Carrera 32 No. 22 - 08, Santa Marta-Colombia \\ (e-mail: jordanguillot@gmail.com; carlosarturo.ing@gmail.com; david.callejasc@hotmail.es) \\ ${ }^{*}$ Autor a quien debe ser enviada la correspondencia
}

Recibido Mar. 31, 2017; Aceptado May. 31, 2017; Versión final Jun. 27, 2017, Publicado Oct. 2017

\begin{abstract}
Resumen
El objetivo de este trabajo es presentar la implementación de un prototipo para la adquisición de señales ambientales, el cual hace parte de un sistema de alerta temprana para gestionar posibles inundaciones en la cuenca del río Manzanares en Santa Marta Colombia. Se diseñó un dispositivo que se encarga de monitorear la humedad del suelo, precipitación y nivel de agua del río en estudio; así como registrar, almacenar y enviar estas variables en tiempo real a través de la red móvil celular. El prototipo responde satisfactoriamente en diferentes condiciones de prueba, adquiriendo las señales ambientales y enviando remotamente al servidor. Con la configuración realizada, los sensores muestran salida estable y capacidad de respuesta a cambios bruscos de humedad del suelo y nivel de agua. El dispositivo de adquisición representa un logro en términos de integración de hardware a bajo costo para la mitigación del riesgo debido a las inundaciones.
\end{abstract}

\section{Environmntal Signals Acquisition for an Early Warning System}

\begin{abstract}
This article presents a prototype of environmental signal acquisition system as part of an early warning system, in order to manage possible floods in the Manzanares river basin in Santa Marta, Colombia. The system aims to monitor the soil moisture, rainfall and water level of the site under study, as well as register, store and send these variables in real time through the cellular network. The prototype responds satisfactorily in different test conditions, acquiring the environmental signals and sending remotely to the server. With the performed configuration, sensors show stable output and good response to sudden changes in soil moisture and water level. The acquisition device represents an achievement in terms of low cost hardware integration for risk mitigation due to flooding.
\end{abstract}

Keywords: acquisition system; early warning system; soil moisture; water level; rainfall 


\section{INTRODUCCIÓN}

Los desastres que producen las inundaciones se presentan como un reto que necesita toda la atención de la comunidad mundial, debido a que este tipo de problemas afectan tanto a países en vía de desarrollo como a los desarrollados. A nivel mundial se ha incrementado el número de eventos de esta naturaleza debido a factores como el cambio climático, deforestación, acumulación de sedimentos, intervención de obras e infraestructura, minería y extracción de recursos a las laderas de ríos; lo cual ha generado la aplicación de diferentes políticas gubernamentales con el fin de generar mecanismos que conlleven a la reducción y mitigación de los riesgos debido a las inundaciones (Préndez y Calderón, 2013; Cools et al., 2016). En esta dirección, es de resaltar la perspectiva holística presentada en (Kundzewicz et al., 2014) sobre los riesgos de inundación debido a las lluvias a nivel mundial, para lo cual utilizaron el informe especial sobre la gestión del riesgo del grupo intergubernamental de expertos sobre el cambio climático. No obstante, la prevención y atención de desastres debido a las inundaciones aún necesita mayor difusión y compromiso de todos los organismos gubernamentales, entidades privadas y poblaciones afectadas.

La situación mundial no es ajena a la que hoy se presenta en Colombia (Bedoya y López, 2015), en particular en la cuenca del río Manzanares que nace en la vertiente noreste de la Sierra Nevada de Santa Marta y se extiende desde el nivel del mar hasta la cota de $2300 \mathrm{msnm}$, con $174.54 \mathrm{~km}^{2}$ de superficie, $70.34 \mathrm{~km}$ de perímetro y $33.50 \mathrm{~km}$ de largo en su cauce principal. Las crecientes súbitas del río Manzanares en época de lluvia ocasionan inundaciones en los barrios ubicados en los sectores bajos. Según la información presentada en (López, 2010; Caracol, 2016), las inundaciones ocasionan daños en los mobiliarios y electrodomésticos de los habitantes, los cuales en muchas ocasiones no tienen tiempo para reaccionar ante las crecientes del río y no logran aplicar las medidas correctivas para poner a salvo sus pertenencias. De igual forma sucede con los organismos de atención que no alcanzan a actuar oportunamente ante este tipo de eventos.

En este contexto surgen un conjunto de mecanismos orientados a disminuir los riesgos por inundaciones, donde los Sistemas de Alerta Temprana (SAT) juegan un papel fundamental debido a que, según (Fakhruddin et al., 2015), presentan un procedimiento diseñado para advertir con la debida anticipación de una amenaza potencial en la población, con el fin de que puedan proteger la vida y sus propiedades. De acuerdo a (Morss et al., 2016), estos mecanismos deben incluir activamente a las comunidades en riesgo, diseminar eficazmente alertas y garantizar una preparación constante.

La adquisición e instalación de sistemas de alerta temprana comerciales requiere de una considerable inversión económica debido a los altos costos de todos los componentes que lo integran, como lo son las estaciones automáticas de medición de nivel, sistemas de comunicación, central de recepción, bases de datos y aplicaciones que le permiten emitir las alertas necesarias a las poblaciones vulnerables. Por esta razón, resulta viable el diseño y la construcción de un sistema de adquisición de señales ambientales con conectividad móvil que minimice los costos y que garantice funcionalidad durante todas las horas del día. De acuerdo a (Jadhav y Satao, 2016), la necesidad de disponer de sistemas de medición que sean robustos, que funcionen sin ningún tipo de interrupción durante largos periodos de tiempo y que además reduzcan las tareas de mantenimiento, ha llevado a que en la actualidad se desarrollen sistemas multisensores que puedan supervisar y controlar dispositivos remotamente mediante la implementación de redes inalámbricas de sensores. En ese sentido, los autores (Rashid y Husain, 2016) presentan aplicaciones e implementaciones de redes de sensores inalámbricas en áreas urbanas.

Diferentes tecnologías son implementadas para la conectividad inalámbrica entre las más importantes se pueden mencionar ZigBee, Wi-Fi, Bluetooth y GPRS. Algunos trabajos que han utilizado este tipo de tecnologías son: (Cheng y Ho, 2016) implementaron una tecnología multicanal en redes de sensores inalámbricos ZigBee; (Batista et al., 2014) realizaron un monitoreo inalámbrico de aerogeneradores urbanos por el protocolo ZigBee y (Samotaev et al., 2014) desarrollaron una matriz de sensores digitales inalámbricos Wi-Fi para monitoreo de gas ambiental. En la misma dirección, (Lian et al., 2013) diseñaron un sistema de control inteligente de múltiples sensores basado en la integración de tecnología innovadora a través de redes ZigBee y Wi-Fi; (Choudhury et al., 2015) implementaron un sistema de adquisición de datos sensoriales basado en redes ZigBee y Bluetooth; (Gutiérrez et al., 2014) realizaron un sistema automatizado de riego que utiliza una red de sensores inalámbricos y un módulo GPRS. Finalmente, (Challoo et al., 2012) presentan una visión general y evaluación de las tecnologías inalámbricas y la coexistencia de dispositivos ZigBee, Bluetooth y Wi-Fi.

Para la realización de este trabajo se utilizó un módulo GSM/GPRS que ofrece la característica de transmitir los datos de manera óptima, cómoda y con una cobertura que está determinada por el alcance del operador de telefonía celular que se utilice. Como sistema de comunicación de respaldo, se implementó una red tipo malla utilizando módulos XBee a partir del estándar Zigbee. En ese sentido, el objetivo de este trabajo está orientado al diseño e implementación de un sistema que garantiza la adquisición, digitalización y transmisión 
inalámbrica a un servidor central de las señales provenientes de los sensores ambientales, con el fin de concebir predicciones de posibles inundaciones en la cuenca del río Manzanares en Santa Marta, Colombia. Se presentan los resultados obtenidos al probar el sistema en un ambiente de prueba que emula las condiciones finales a las que será expuesto en la cuenca del río Manzanares.

\section{METODOLOGÍA}

En la Figura 1 se muestra el diagrama de bloques del SAT. El elemento central del sistema de adquisición es un microcontrolador ATmega 2560, el cual fue seleccionado teniendo en cuenta la disponibilidad de entradas y salidas digitales para la comunicación SDI 12, puerto serie USART para conectarse al módulo de comunicación inalámbrica y posibilidad de recibir interrupciones externas, entre otras.

Adicionalmente, se tuvo en cuenta que es un dispositivo sencillo de programar, fiable y con alta documentación, el cual viene compacto en la plataforma de bajo costo Arduino, facilitando las herramientas de conexión entre módulos (Reguera et al., 2015). El microcontrolador se encarga de la adquisición de las señales analógicas que se digitalizan y se entregan al dispositivo de comunicación inalámbrica para ser enviadas al servidor; además de gestionar y controlar cada operación funcional de los módulos presentados en la Figura 1. El sistema integra una memoria del tipo Micro SD, un reloj de tiempo real (RTC), una interfaz que permite al usuario visualizar el estado de las variables con hora, fecha y configurar los tiempos en los que el sistema debe ejecutar las operaciones establecidas, sensores de humedad del suelo, nivel, precipitación, módulo GSM/GPRS para la transmisión de los datos al servidor y XBee de respaldo.

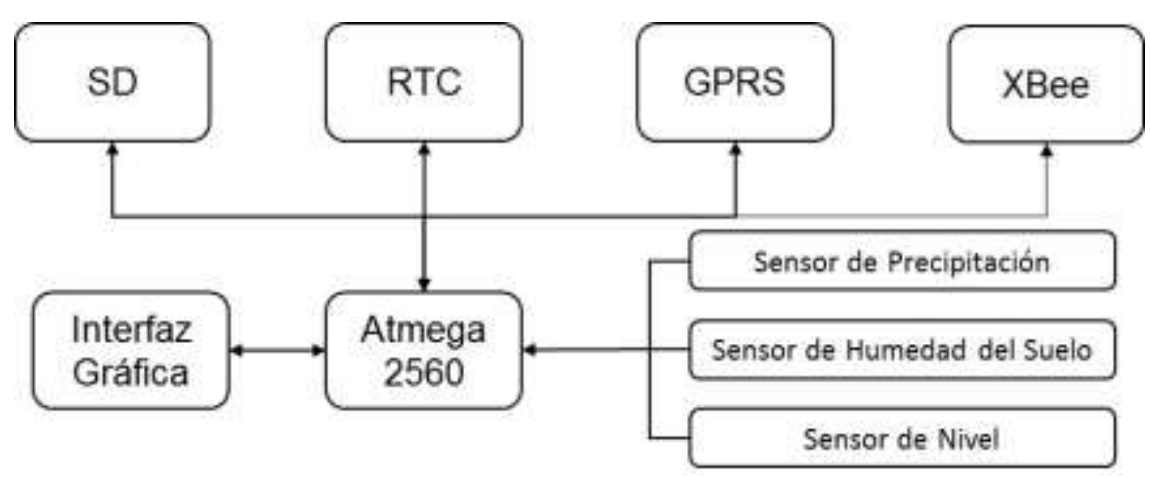

Fig. 1: Diagrama de bloques del SAT

\section{Módulo SD}

Se utilizó un módulo SD Card Shield para el almacenamiento de variables con fecha y hora en sitio, el cual se comunica con el Arduino mega a través del protocolo SPI permitiendo leer y escribir datos en diferentes archivos, siempre y cuando se cierre el último archivo utilizado. Esto brinda la posibilidad de separar la información proveniente de los diferentes sensores. Con este módulo se incorpora la capacidad de Datalogger al sistema de adquisición y procesamiento de las señales, puesto que permite almacenar las variables en una memoria MicroSD de $8 \mathrm{~GB}$, permitiendo acceder a los archivos desde bloc de notas o importarlo desde Microsoft Excel.

\section{Módulo RTC}

Se utilizó un RTC I2C para obtener la fecha y hora actual. Este módulo incorpora un reloj en tiempo real DS1307, una batería de alimentación en caso de ausencia de energía, una pequeña memoria EEPROM para memorizar los datos y un bus I2C para la comunicación con el Arduino mega. La fecha y la hora suministrada son enviadas al servidor paralelamente al estado actual de cada medición ambiental.

\section{Módulo GSM/GPRS}

Se utilizó el módulo GSM/GPRS Sim900 para la transmisión de los datos, el cual permite enviar y recibir llamadas, mensajes o conexión a internet, proporcionando la condición de teléfono móvil al proyecto. Estas bondades están sujetas al uso de una Sim Card activa en un operador de telefonía móvil. Esta tecnología ofrece al sistema de adquisición las herramientas para operar en zonas remotas de la mano del operador de señal móvil funcional en sitio. Con este módulo se envían los datos de los sensores a una aplicación móvil que fue desarrollada en una segunda fase del proyecto, la cual a través de un modelo de predicción de inundaciones emite tres tipos de alerta: amarilla, naranja y roja. Estas alertas son visibles tanto por los 
miembros de la comunidad y las entidades pertenecientes al centro de operaciones de emergencia del distrito de Santa Marta. Adicionalmente, este módulo permite el envío de los niveles del río vía mensajes de texto.

\section{Diseño del Protocolo de Comunicación Inalámbrica GSM/GPRS}

Los intervalos de envío de las diferentes variables se ejecutan dependiendo el tiempo configurado por el usuario en la interfaz gráfica, cuando esta condición se cumple el sistema crea una cadena de caracteres con la información precisa de cada variable (Id, Fecha, Hora y Magnitud) y las envía por el puerto serie al módulo GSM/GPRS utilizando comandos AT. En la Figura 2 se observa la configuración inicial del módulo GSM/GPRS, donde primero se establece el perfil de conexión, luego se establece el APN del operador móvil utilizado, se abre un contexto GPRS y por último se inicializa una conexión HTTP.

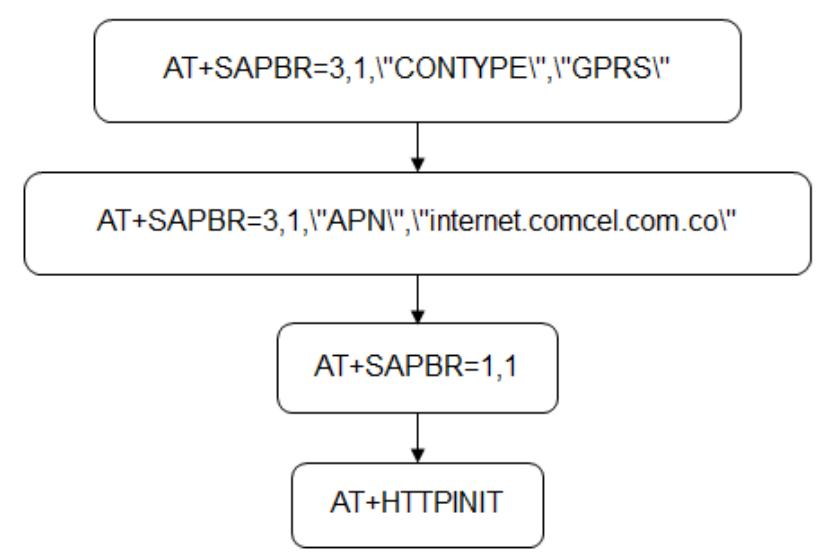

Fig. 2: Configuración inicial de módulo GSM/GPRS

La Figura 3 muestra la secuencia de comandos para enviar la trama de datos a través de la red móvil. Se establece la dirección URL del servidor donde se almacenarán los datos, concatenando la información pertinente: Id, variable ambiental, hora y fecha. Luego se envían los datos y se espera la respuesta del servidor, por último se envía un espacio con el fin de limpiar el buffer del módulo. Si el servidor no responde o suministra una respuesta de fallo, el sistema intenta enviar el mensaje dos veces más, en caso de persistir el error reinicia y configura el módulo para iniciar una nueva conexión.

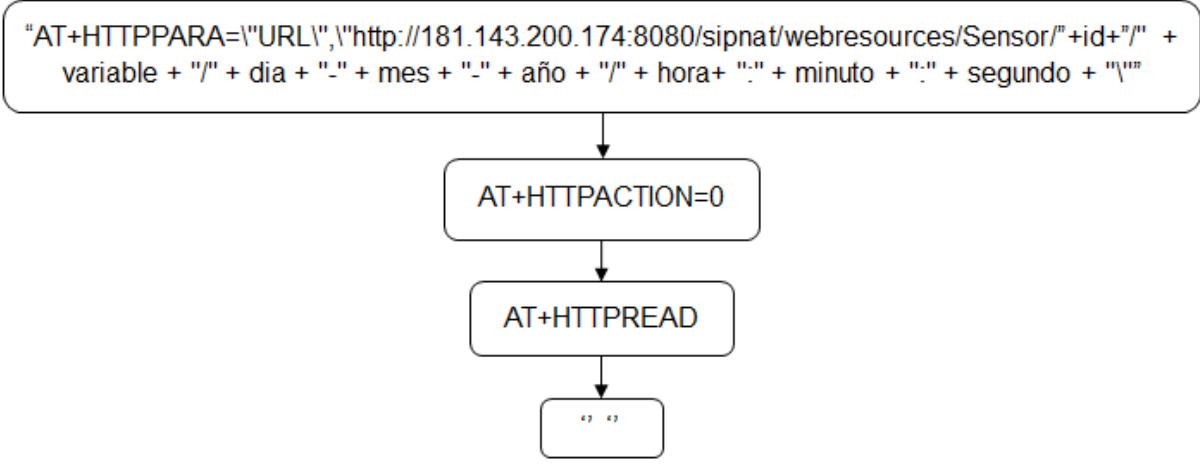

Fig. 3: Envío de trama de datos

Red Mesh con XBee

Como sistema de respaldo para la transmisión inalámbrica de los datos, se implementó una red mesh estándar basada en el protocolo Digimesh, la cual cuenta con un coordinador de red, dos enrutadores de red y un nodo final. En todos los puntos se utilizaron los módulos XBee PRO de $900 \mathrm{MHz}$ con conector RPSMA; los cuales permiten un alcance de hasta $14 \mathrm{Km}$. Para cada uno de los nodos se utilizaron antenas de alta ganancia de 10DBi. Esta red tipo malla permite transmitir las señales ambientales adquiridas en caso de que se presenten problemas con la comunicación GSM/GPRS.

\section{Protocolo de Adquisición de Señales de Humedad del Suelo y Nivel.}

Se optó por implementar sensores basados en el protocolo SDI-12, puesto que incluyen procesadores embebidos donde almacenan toda la información de fábrica para que el usuario pueda disponer de esta por 
medio de comandos AT. Inicialmente los sensores tienen una dirección definida de fábrica que es la cero, por tanto es necesario cambiarla a través del comando "aAb!", donde "a" representa la dirección anterior y "b" la dirección nueva. Una vez asignadas las direcciones de cada sensor, se procede a enviarle el comando de inicio de medición "aM!", donde "a" es la dirección del sensor. Después de un tiempo de espera se envía el comando de solicitud de medición "aD0!", donde "a" es la dirección del sensor utilizado. Otras de las razones por las que fue utilizada esta tecnología tienen que ver con el bus de transmisión serial y el ahorro de hardware que estos sensores brindan al sistema, puesto que sobre la misma línea de datos se pueden conectar múltiples dispositivos SDI-12.

\section{Sensor de Humedad del Suelo ADCON SM1}

Consiste en un sistema flexible de medición de humedad del suelo basado en capacitancia, con una longitud de $30 \mathrm{~cm}$ y posee 3 dispositivos de medición en intervalos de $10 \mathrm{~cm}$. Este sensor es instalado en el suelo dentro de un tubo PVC sellado de $32 \mathrm{~mm}$ de diámetro. La esfera de influencia de los sensores es de aproximadamente $10 \mathrm{~cm}$ de radio alrededor del tubo. Este sensor mide el contenido de agua volumétrica con una precisión de $\pm 2 \%$ y cuenta con calibración de fábrica de $\pm 1 \%$. Durante la fase de configuración se le asignó la dirección uno a este sensor. En la Figura 4 se observa el diagrama de flujo para solicitar las mediciones que realiza. Por recomendaciones del fabricante se fijó un tiempo de espera de aproximadamente 10 segundos entre comandos, para que el sensor pueda tomar las muestras necesarias.

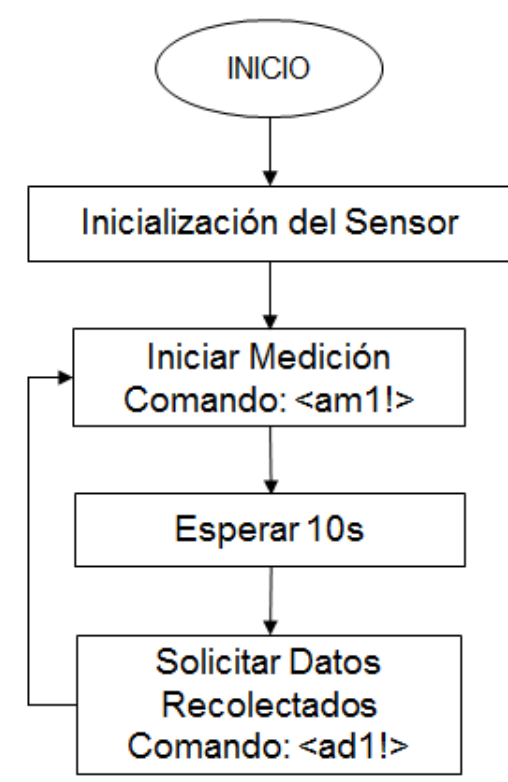

Fig. 4: Diagrama de flujo de adquisición de la humedad del suelo

\section{Sensor de Nivel OTT RLS}

El sensor por radar OTT RLS sirve para medir, sin contacto físico directo, niveles de aguas superficiales. Su principio de funcionamiento se soporta en la tecnología de radar por impulsos, en la cual la antena emisora envía breves impulsos de radar con una frecuencia de $24.1 \mathrm{GHz}$. La antena receptora recibe estos impulsos reflejados en la superficie del agua y calcula a partir de ahí la distancia entre el sensor y la superficie. El sensor no necesita labores de ajuste o calibración y no cuenta con piezas que deban ser reemplazadas regularmente. El error de medición cuando se trabaja con el protocolo SDI-12 oscila entre $\pm 3 \mathrm{~mm}$ y $\pm 10 \mathrm{~mm}$, el cual varía dependiendo de la distancia a la que se instale el sensor. El diámetro de medición del lóbulo del sensor varía entre $1.06 \mathrm{~m}$ y $7.44 \mathrm{~m}$, lo cual depende de la distancia entre el sensor y la superficie del agua. La programación de este sensor es similar a la realizada para el sensor de humedad con la única diferencia en el tiempo de espera (20s) y dirección de los sensores (0).

\section{Sensor de Precipitación Young 52202}

Se encarga de medir la cantidad de precipitación en una superficie de $200 \mathrm{~cm}^{2}$ con una resolución de $0.1 \mathrm{~mm}$. Su funcionamiento se basa en un sistema de báscula, el cual está conectado a un contador donde cada pulso representa una dimensión de lámina de agua de $0.1 \mathrm{~mm}$ que equivale a 1 litro/ $\mathrm{m}^{2}$ de superficie. En la Figura 5 se puede observar en detalle el sistema de báscula. 


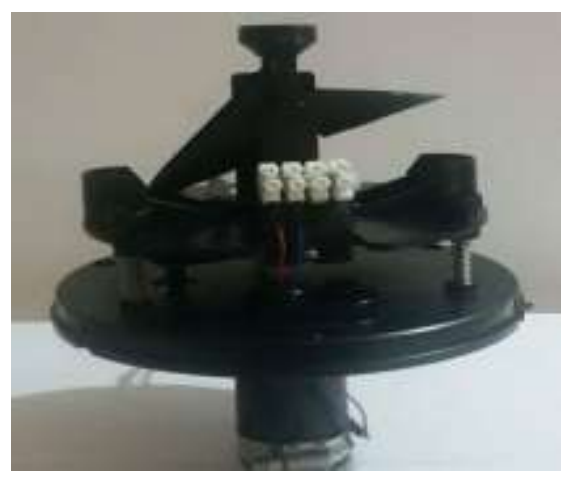

Fig. 5: Sistema de báscula

Este sensor requiere una calibración periódica cada 12 meses según lo que recomienda el fabricante. El proceso se realiza con el pluviómetro correctamente nivelado y aplicando $10 \mathrm{ml}$ por minuto en el embudo de recolección. El cubo se debe inclinar cinco veces por cada $10 \mathrm{ml}$ de agua. En el caso que se apliquen $100 \mathrm{ml}$, el sensor debe entregar un recuento de $50 \pm 1$. Si el recuento muestra un error superior al $2 \%$, el sensor dispone de dos tornillos de calibración que se deben ajustar al tiempo. Cuando la cuenta es menor de lo esperado, los tornillos se deben levantar; en el caso contrario se deben bajar los tornillos. Antes de utilizar el sensor, se aplicó el procedimiento anterior para verificar la correcta calibración. Los intervalos de adquisición de precipitación son establecidos de acuerdo a las necesidades del usuario, por ende, estos tiempos son configurados por medio de la interfaz de tiempos del datalogger. En la Figura 6 se muestra el diagrama de flujo de la adquisición de datos de precipitación.

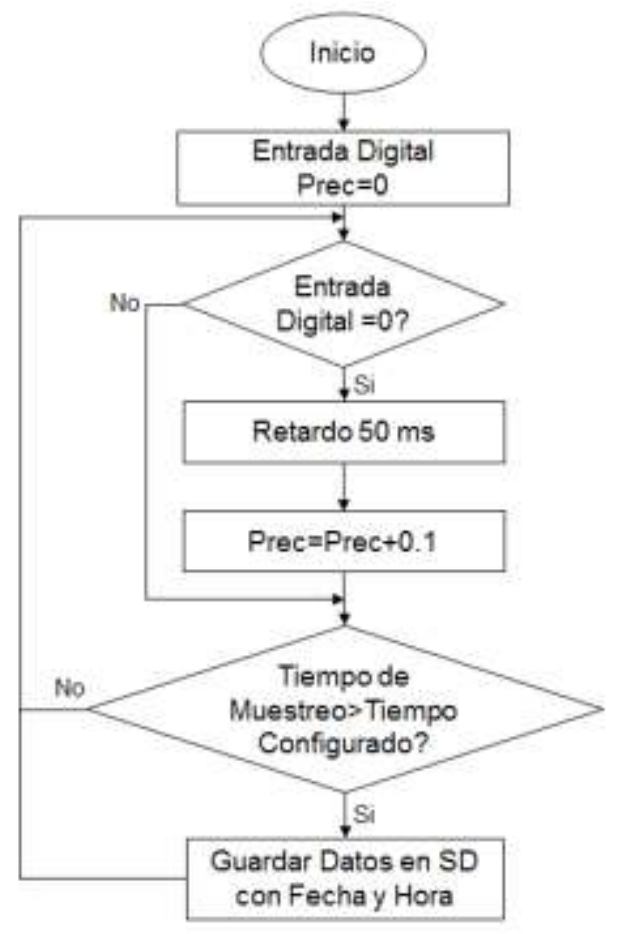

Fig. 6: Diagrama de flujo de adquisición de precipitación

En la Figura 7 se muestra el sensor de humedad del suelo ADCOM SM1, sensor de nivel OTT RLS y sensor de precipitación Young 52202 acoplados al tablero electrónico (Datalogger) que se encarga de procesar toda la información y transmitirla de forma inalámbrica. Todos los equipos están instalados en un ambiente de prueba que permite evaluar el desempeño global del sistema para una posterior instalación en la cuenca del río Manzanares.

\section{Interfaz gráfica LCD táctil TFT 3.5}

La interfaz gráfica se encarga de la interacción entre el usuario y el sistema incluyendo cualidades como visualización de variables, fecha, hora y un panel de configuración de intervalos de tiempos de adquisición y almacenamiento. La Figura 8, recuadro (a), muestra la pantalla principal del sistema donde se visualizan los datos de interés para el usuario. En la parte izquierda están las variables de nivel, humedad del suelo y 
precipitación. En la parte derecha superior se observa la hora y la fecha en tiempo real, mientras que en la parte derecha inferior se muestra el estado de la conectividad inalámbrica.

En la parte inferior izquierda se observan dos iconos: una flecha para retroceder y un botón de ajustes para ingresar a la segunda pantalla. En el recuadro (b) se muestra el panel de configuración de intervalos de tiempos, conformado por un teclado numérico, un botón de aceptar (verde), un botón de borrar (rojo) y las 4 variables a configurar con sus respectivos datos almacenados en la EEPROM.

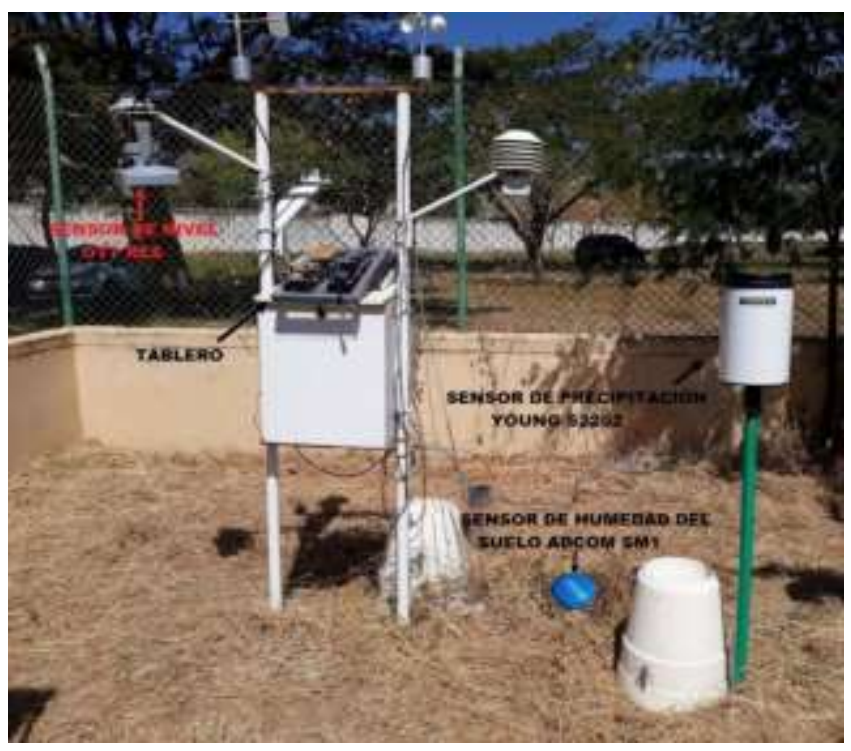

Fig. 7: Sistema de adquisición

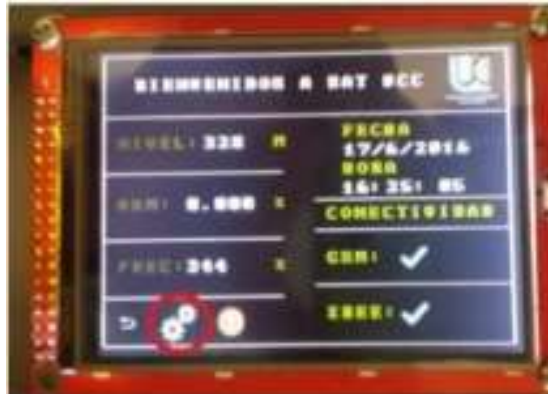

(a)

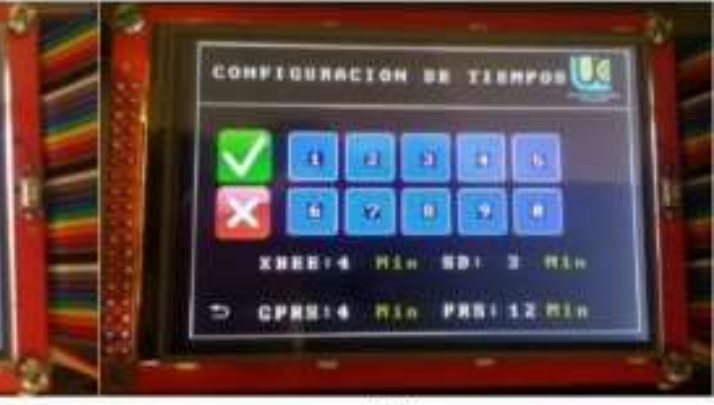

(b)

Fig. 8: (a) Pantalla principal; (b) configuración

\section{RESULTADOS Y DISCUSIÓN}

Los resultados arrojados en esta investigación están basados en la adquisición de las señales ambientales y la transmisión inalámbrica de las mismas. El sensor de humedad del suelo se instaló a $30 \mathrm{~cm}$ de profundidad. Se realizaron 3 pruebas en diferentes condiciones de humedad con un tiempo de muestreo de 2 horas.

En la prueba 1 se capturaron los datos sin ninguna perturbación externa, obteniendo como resultado una respuesta constante con pequeñas variaciones. En la prueba 2 se perturbó la condición ambiental con 0.5 litros de agua, lo cual arrojó un incremento de la humedad del suelo manteniéndose las características de linealidad. Después de unos minutos los valores se estabilizan a un ritmo casi constante. La prueba 3 consistió en capturar los valores de humedad del suelo arrojados después de 48 horas de haber realizado la prueba 2 , lo cual dio como resultado una disminución de los valores de humedad del suelo, quedando finalmente a un nivel constante. Cabe agregar que el área de tierra experimental no fue expuesta de manera abrupta a los rayos del sol. En la Figura 9 se muestra en detalle los resultados obtenidos para los 3 casos.

El pluviómetro fue sometido a 5 pruebas, las cuales consistieron en exponerlo a distintas cantidades de precipitación, con el fin de observar su respuesta en diversas condiciones. Se utilizaron perturbaciones que oscilaron entre 100 y $500 \mathrm{cc}$ de agua que fueron aplicadas en diferentes instantes de tiempo y realizando cambios dinámicos en la cantidad de líquido utilizada. En la Tabla 1 se muestran los resultados obtenidos para los 5 escenarios, en los cuales se evidencia la respuesta proporcional del sensor de acuerdo a la cantidad de cc de agua aplicada. 
Tabla 1: Respuesta del sensor de precipitación.

\begin{tabular}{|c|c|c|c|c|}
\hline Prueba & $\begin{array}{c}\text { Precipitación } \\
(\mathrm{cc})\end{array}$ & $\begin{array}{c}\text { Nivel de Lámina } \\
\text { de Agua }(\mathrm{mm})\end{array}$ & $\begin{array}{c}\text { Cambios de } \\
\text { Estado Báscula }\end{array}$ & $\begin{array}{c}\text { Tiempo de Respuesta } \\
\text { del Sensor }(\mathrm{s})\end{array}$ \\
\hline 1 & 100 & 2.5 & 25 & 5 \\
\hline 2 & 400 & 10.8 & 108 & 20 \\
\hline 3 & 300 & 8.4 & 84 & 15 \\
\hline 4 & 500 & 13.1 & 131 & 25 \\
\hline 5 & 200 & 6.6 & 66 & 10 \\
\hline
\end{tabular}

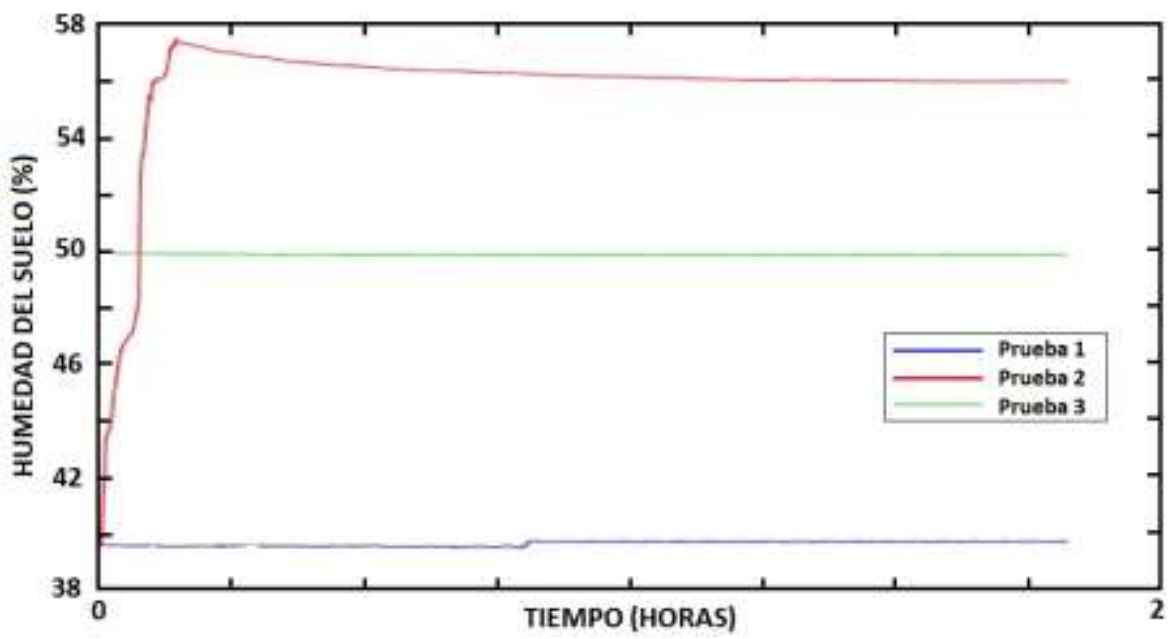

Fig. 9: Respuesta del sensor de humedad del suelo

En relación a los resultados obtenidos con el sensor de nivel, es de destacar que se obtuvieron valores exactos en las mediciones de distancias, realizando diferentes pruebas en varias superficies entre ellas concreto, cartón y agua. El sensor tiene un máximo de detección de 35 metros y un punto ciego de cero a cuatro centímetros. El proceso de acople de los módulos mencionados anteriormente dio como resultado el tablero electrónico del sistema de adquisición de señales móvil de la Figura 10, en el cual se observa el SAT, módulo GSM/GPRS y el XBee coordinador utilizado en la estación de mediciones.

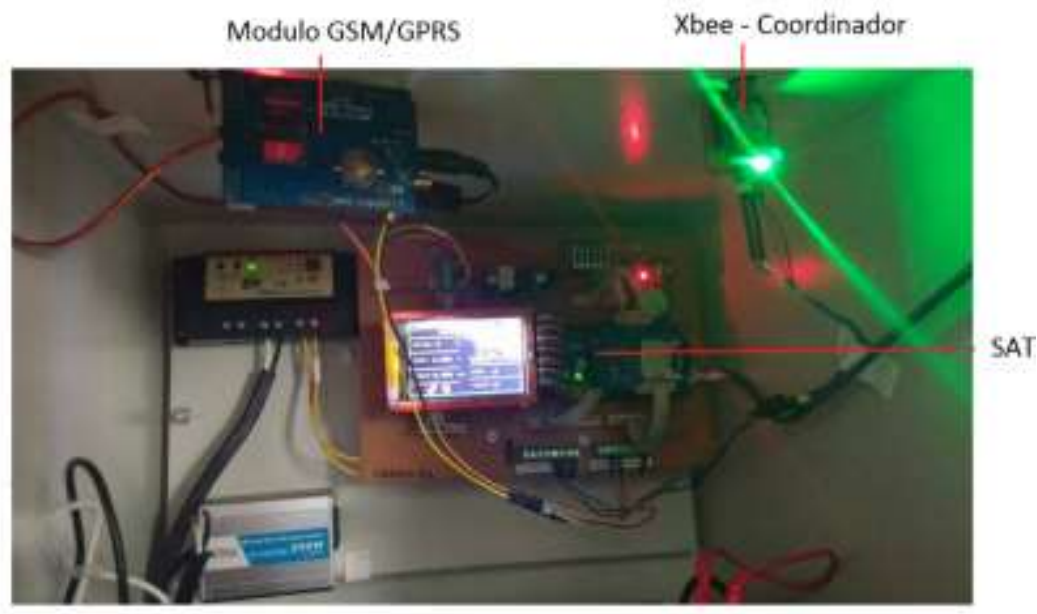

Fig. 10: Tablero electrónico del sistema de adquisición de señales móvil (Datalogger)

Los resultados de este trabajo pueden ser contrastados con lo realizado por (Do et al., 2015), quienes en la primera fase de su investigación desarrollaron una estación de medición compuesta por un módulo GSM/GPRS SIM900, sensor de medición de nivel de agua y sensor de precipitación. Al igual que en la presente investigación, se crearon condiciones para evaluar el sistema en un ambiente de prueba. Los datos de los dos sensores fueron almacenados en un módulo de $2 \mathrm{~GB}$, con un tiempo de muestreo de 10 minutos con lo cual obtuvieron capacidad de almacenamiento para 437 días. En contraste, en esta investigación se utilizó una memoria de $8 \mathrm{~GB}$, la cual brinda una capacidad de almacenamiento de 58 días utilizando el mismo periodo de muestreo; pero almacenando tres variables. Por otra parte, el sensor de precipitación fue evaluado en un periodo de tiempo de 80 minutos ante cambios abruptos, demostrando una respuesta lineal. 
En el mismo sentido, es de mencionar el trabajo realizado por (Hernández et al., 2016) en la implementación de un medidor de nivel de agua con el objetivo final de alertar a una población ante amenaza de inundaciones. Al igual que en este trabajo, se simula un ambiente de prueba con un proyecto piloto compuesto por el sensor de nivel y el dispositivo de adquisición basado en la herramienta de hardware libre Netduino. La principal diferencia con el presente trabajo radica en que se utilizó la tecnología WiFi para la transmisión de los datos a un servidor web. Los resultados demostraron la flexibilidad de la herramienta Netduino para adquirir la señal del sensor de acuerdo a tres niveles preestablecidos. Adicionalmente, la plataforma desarrollada permite almacenar los datos medidos en una memoria externa; aunque esta funcionalidad no fue evaluada.

Por su parte, en (Asmara y Aziz, 2011) realizaron el proceso de adquisición de tres sensores de nivel con un microcontrolador de gama alta PIC18F452 y un módulo GSM para la transmisión de los datos. El sistema fue evaluado y se verificó su correcto funcionamiento en diferentes ambientes de pruebas en estanques de agua; extrayendo como principal conclusión la pertinencia de utilizar el prototipo para futuras instalaciones en las orillas de los ríos y sistemas de drenaje. Los sensores de nivel mostraron una respuesta lineal y cada detección de nivel se representó con un voltaje de $5 \mathrm{~V}$. Es de resaltar que en este trabajo los autores no realizaron un proceso de almacenamiento de datos puesto que el microcontrolador se utilizó como dispositivo de procesamiento y de enlace con el módulo GSM para enviar mensajes de texto a un dispositivo móvil. En la misma dirección, el trabajo realizado por (Islam et al., 2014) se centró en la adquisición del nivel del agua con un sensor Honeywell LL103101 y un microcontrolador. El sistema fue evaluado en un estanque de prueba con el cual se obtuvieron los datos del sensor de nivel en un periodo de observación de 4 horas los cuales fueron enviados directamente a un servidor web utilizando una tarjeta ethernet.

\section{CONCLUSIONES}

A partir del prototipo desarrollado para la adquisición de señales ambientales y de los resultados obtenidos a partir de las pruebas realizadas, se derivan las siguientes conclusiones:

1.- El sistema implementado cumple con las condiciones de captura, procesamiento, almacenamiento y transmisión de los parámetros ambientales, dando por hecho la característica de un sistema autónomo y robusto, basado en la utilización de herramientas de hardware libre Arduino.

2.- La elección de trabajar con sensores basados en el protocolo SDI-12 ofreció la posibilidad de trabajar de manera cómoda y precisa, en virtud de que cada sensor se encarga de tomar varias muestras de la variable y realizar un promedio de las mismas. Además, para proyecciones futuras esta tecnología ofrece la ventaja de ampliar la red de dispositivos sin necesidad de incrementar el hardware; sólo es necesario una línea de datos para comunicarse con todos los sensores.

3.- Las pruebas de conectividad en sitio permitieron determinar que operador móvil funcionaba de manera estable en el lugar de instalación, el sistema envió al servidor de manera exitosa los datos del módulo de adquisición con la estampa de hora y fecha actual.

4.- Una vez analizado el funcionamiento del sistema y haberlo sometido a distintas pruebas en tiempo real, se obtuvieron los resultados esperados, proporcionándole la información ambiental necesaria al servidor web encargado de gestionar los futuros algoritmos de predicción para emitir las alertas.

\section{AGRADECIMIENTOS}

Los autores agradecen al Departamento Administrativo de Ciencia, Tecnología e Innovación de Colombia (Colciencias) por la financiación del proyecto Sistema de Alerta Temprana para Gestionar el Riesgo por Inundación en la Cuenca del Río Manzanares, con recursos del patrimonio autónomo fondo nacional de financiamiento para la ciencia, la tecnología y la innovación, Francisco José de Caldas.

\section{REFERENCIAS}

Asmara, W. y Aziz, N., SMS flood alert system, IEEE Control and System Graduate Research Colloquium, 1822, Shah Alam-Malaysia, 27 a 28 de Junio (2011)

Batista, N.C., Melicio, R., Mendes, V.M. y Figueiredo, J., Wireless Monitoring of Urban Wind Turbines by ZigBee Protocol: Support Application Software and Sensor Modules, doi: 10.1016/j.protcy.2014.10.182, Procedia Technol., (en línea), 17, 461-470 (2014)

Bedoya, V. y López, J., Modelo para el Control de Inundaciones durante el Fenómeno De "La Niña" Utilizando un Embalse Hidroeléctrico, doi: 10.4067/S0718-07642015000200011, Información Tecnológica, (en línea), 26(2), 89-100 (2015) 
Caracol, Fuerte lluvia causa estragos en Santa Marta (en la web: https://goo.gl/4xheb2, acceso: 13 de junio 2017), Caracol Radio (2016)

Challoo, R., Oladeinde, A., Yilmazer, N., Ozcelik, S. y Challoo, L., An Overview and Assessment of Wireless Technologies and Co-existence of ZigBee, Bluetooth and Wi-Fi Devices, doi: 10.1016/j.procs.2012.09.091, Procedia Comput Sci, (en línea), 12, 386-391 (2012)

Cheng, C.H. y Ho, C.C., Implementation of multi-channel technology in ZigBee wireless sensor networks, doi: 10.1016/j.compeleceng.2015.10.002, Comput \& Electr Eng, (en línea), 56, 498-508 (2016)

Choudhury, S., Kuchhal, P., Rajesh, S. y Anita, ZigBee and Bluetooth Network based Sensory Data Acquisition System, doi: 10.1016/j.procs.2015.04.195, Procedia Comput Sci, (en línea), 48, 367-372 (2015)

Cools, J., Innocenti, D. y O'Brien, S., Lessons from flood early warning systems, doi: 10.1016/j.envsci.2016.01.006, Environ Sci \& Policy, (en línea), 58, 117-122 (2016)

Do, H., Vo, M., Tran, V., Tan, P. y Trinh, C., An early flood detection system using mobile networks, International Conference on Advanced Technologies for Communications (ATC), 599-603, Ho Chi MinhVietnam, 14 a 16 de Septiembre (2015)

Fakhruddin, S.H., Kawasaki, A. y Babel, M.S., Community responses to flood early warning system: Case study in Kaijuri Union, Bangladesh, doi: 10.1016/j.ijdrr.2015.08.004, Int. J. Disaster Risk Reduction, (en línea), 14(4), 323-331 (2015)

Gutiérrez, J., Villa, J.F., Nieto, A. y Porta, M.A., Automated Irrigation System Using a Wireless Sensor Network and GPRS Module, doi: 10.1109/TIM.2013.2276487, IEEE T Instrum Meas, (en línea), 63(1), 166-176 (2014)

Hernández, J., Ovando, M., Acosta, F. y Pancardo, P., Water Level Meter for Alerting Population about Floods, IEEE 30th International Conference on Advanced Information Networking and Applications (AINA), 879-884, Crans-Montana, 23 a 25 de Marzo (2016)

Islam, M., Islam, T., Syrus, M. y Ahmed, N., Implementation of flash flood monitoring system based on wireless sensor network in Bangladesh, International Conference on Informatics, Electronics \& Vision (ICIEV), 1-6, Dhaka-Bangladesh, 23 a 24 de Mayo (2014)

Jadhav, P. y Satao, R., A Survey on Opportunistic Routing Protocols for Wireless Sensor Networks, doi: 10.1016/j.procs.2016.03.076, Procedia Comput Sci, (en línea), 79, 603-609 (2016)

Kundzewicz, Z., Kanae, S. y otros 15 autores, Flood risk and climate change: global and regional perspectives, doi: 10.1080/02626667.2013.857411, Hydrological Sciences Journal, (en línea), 59(1), 1-28 (2014)

Lian, K.Y., Hsiao, S.J. y Sung, W.T., Intelligent multi-sensor control system based on innovative technology integration via ZigBee and Wi-Fi networks, doi: 10.1016/j.jnca.2012.12.012, Journal of Network and Computer Applications, (en línea), 36(2), 756-767 (2013)

López, O., Decenas de familias pasaron la noche en vela por desbordamiento del río Manzanares (en la web: https://goo.gl/WvfVsJ, acceso: 11 de junio 2017), Periódico el Informador (2010)

Morss, R.E., Mulder, K.J., Lazo, J.K. y Demuth, J.L., How do people perceive, understand, and anticipate responding to flash flood risks and warnings? Results from a public survey in Boulder, Colorado, U.S.A., doi: 10.1016/j.jhydrol.2015.11.047, Journal of Hydrology, (en línea), 541, 649-664 (2016)

Préndez, M. y Calderón, V., Análisis de Contaminantes en la Cuenca del Río Aconcagua en Chile. Evaluación de Riesgo Humano y Ambiental, doi: 10.4067/S0718-07642013000100002, Información Tecnológica, (en línea), 24(1), 3-14 (2013)

Rashid, B. y Husain, M., Applications of wireless sensor networks for urban areas: A survey, doi: 10.1016/j.jnca.2015.09.008, Journal of Network and Computer Applications, (en línea), 60, 192-219 (2016)

Reguera, P., García, D., Dominguez, M., Prada, M.A. y Alonso, S., A low-cost open source hardware in control education. Case study: Arduino-feedback MS-150, doi: 0.1016/j.ifacol.2015.11.223, IFAC-PapersOnLine, (en línea), 48(29), 117-122 (2015)

Samotaev, N., Ivanova, A., Oblov, K., Soloviev, S. y Vasiliev, A., Wi-Fi Wireless Digital Sensor Matrix for Environmental Gas Monitoring, doi: 10.1016/j.proeng.2014.11.684, Procedia Eng., (en línea), 87, 1294-1297 (2014) 